PHYSICAL REVIEW E 80, 026101 (2009)

\title{
Self-similarity of waiting times in fracture systems
}

\author{
G. Niccolini \\ Istituto Nazionale di Ricerca Metrologica, Strada delle Cacce 91, 10135 Turin, Italy \\ F. Bosia \\ Department of Physics, Università di Torino, Via Giuria 1, 10125 Turin, Italy
}

A. Carpinteri, G. Lacidogna, A. Manuello, and N. Pugno

Department of Structural Engineering, Politecnico di Torino, Corso Duca degli Abruzzi 24, 10129 Torino, Italy

(Received 6 February 2009; published 3 August 2009)

\begin{abstract}
Experimental and numerical results are presented for a fracture experiment carried out on a fiber-reinforced element under flexural loading, and a statistical analysis is performed for acoustic emission waiting-time distributions. By an optimization procedure, a recently proposed scaling law describing these distributions for different event magnitude scales is confirmed by both experimental and numerical data, thus reinforcing the idea that fracture of heterogeneous materials has scaling properties similar to those found for earthquakes. Analysis of the different scaling parameters obtained for experimental and numerical data leads us to formulate the hypothesis that the type of scaling function obtained depends on the level of correlation among fracture events in the system.
\end{abstract}

DOI: 10.1103/PhysRevE.80.026101

PACS number(s): 89.75.Da, 62.20.M-, 91.55.Jk, 91.30.Dk

\section{INTRODUCTION}

The brittle fracture of materials is a complex phenomenon which occurs according to two broadly defined scenarios. In the first one, failure occurs by sudden propagation of a single fracture without appreciable precursors [1]. In the second, failure occurs as the culmination of progressive damage. Examples include decohesion between inclusions, accumulation of dislocations, debonding between fibers and matrix in composite materials, etc. [2-4]. In particular, acoustic emission (AE) due to microcrack growth precedes the macroscopic failure of fiber composites, rocks, and concrete under stress [5]. The observed power-law scaling for temporal [6], spatial $[7,8]$, and magnitude distributions $[5,9,10]$ of AE events led statistical physicists to consider brittle fracture in analogy to critical-point phenomena, where fracture energy and ultimate stress play the role of critical parameters.

Power-law and critical exponents are not the only scaling predictions for physical systems close to their critical point. Equations of state in the form of scaling laws can be established as well, where the fulfillment of scaling laws is illustrated by data collapse [11]. In the case of fracture phenomena, data collapse was first observed by Bak et al. [12] for the probability densities of waiting times between earthquakes, obtained for different values of minimum threshold magnitude. They carried out a spatiotemporal analysis of seismicity in California in which the waiting-time density was related to the Gutenberg-Richter (GR) law for the distribution of earthquake magnitudes and to the fractal distribution of epicenters into a unified scaling law.

In the following, we analyze the waiting times between AE events in a time series of a new laboratory-scale fracture experiment on a fiber-reinforced concrete (FRC) beam subjected to three-point bending test [13]. The data collapse is obtained on the basis of a different approach introduced by Corral $[14,15]$, recently applied to Italian seismicity (see Fig. 1) [13] and to laboratory rock fracture [16], in which $\mathrm{AE}$ events are characterized only by their occurrence time and their magnitude, while the positions of hypocenters are taken into account solely in the correction for attenuation effects to calculate the magnitudes.

Since the works of Mogi and Scholz [17,18] on AE, we know that the Gutenberg-Richter empirical law can be observed at the laboratory sample scale. They showed that a significant overlap exists between the definition of $\mathrm{AE}$ and earthquake, respectively. This is further reinforced by the

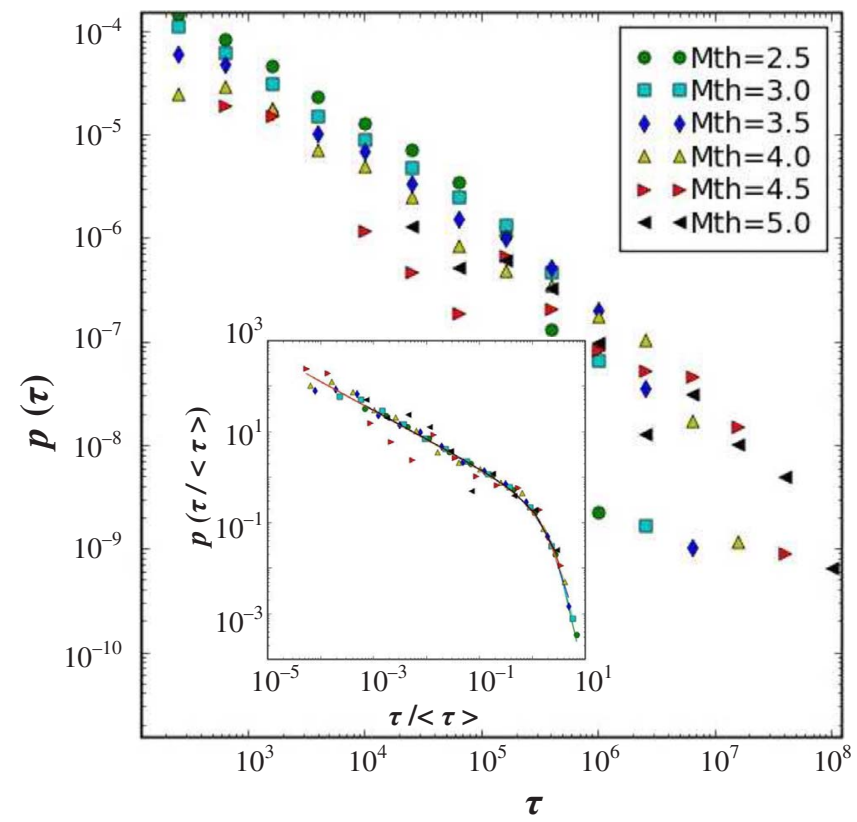

FIG. 1. (Color online) Probability densities of waiting times $\tau$ (measured in seconds) of Italian earthquakes with magnitude $\geq M_{t h}$ during the period 1984-2002, for $M_{t h}$ ranging from 2.5 to 5 . Inset: probability densities of the normalized waiting times $\tau /\langle\tau\rangle$. The data collapse is the signature of scaling behavior (from [13]). 
evidence that brittle fracture obeys similar statistics from tectonic earthquakes to the dislocation movement smaller than the micron size [19]. The analogy between earthquakes and acoustic emissions emphasized in the literature [13-15] relies on the fact that these two phenomena share the same source mechanism: they both are elastic energy released by growing cracks inside a medium. Because of this and the similarity in their statistical behavior, series of AE events can be considered analogous to earthquake sequences despite the different frequency domains (the ultrasonic range of acoustic emissions and the sonic range of the seismic roar). The followed approach regards earthquakes and acoustic emissions as points in space, time (i.e., the occurrence time), and magnitude, without focusing on the frequency content of the events. The goal of such parallelism is to investigate if the results presented for earthquakes are representative of fracture processes at a much smaller scale, i.e., microcracks in a laboratory specimen $[13,15]$.

\section{WAITING-TIME DATA COLLAPSE FOR EARTHQUAKES AND AES}

Traditionally, the most important phenomenological law describing the scaling in fracture systems is the GR law [20], valid both for AEs and earthquakes [9,16,20,21]. The GR law determines the number $N\left(M_{t h}\right)$ of events with magnitude $M \geq M_{\text {th }}$ occurring in a specified region and during a sufficiently long period of time $T$,

$$
N\left(M_{t h}\right)=10^{a-b M_{t h},}
$$

where $a$ and $b$ are constants. The quantity $10^{a}$ is the hypothetical number of events in the region with a magnitude greater than 0 , while $b$, or " $b$ value," determines the number of events with a given magnitude. Typically, the seismic $b$ value depends on the region under study, while the acoustic $b$ value on the damage level reached in the material.

In the case of AE monitoring tests, the region is the volume of the monitored specimen and $T$ is the time duration of the test. For each $M_{t h}$ value (equivalent to a voltage threshold $V_{t h}$ if we consider the AE amplitudes), all the AE events with $M \geq M_{t h}$ define a point process in time where events occur at $t_{i}$ with $1 \leq i \leq N\left(M_{t h}\right)$, and therefore, the time intervals between consecutive events - already referred to as waiting times - can be obtained as $\tau_{i} \equiv t_{i}-t_{i-1}$. If we focus on the temporal properties of this process, the mean waiting time, $\langle\tau\rangle_{M_{t h}} \equiv T /\left[N\left(M_{t h}\right)-1\right]$, is the only information provided by the GR law,

$$
\langle\tau\rangle_{M_{t h}}=T 10^{-a+b M_{t h}} .
$$

Since waiting times are broadly distributed, the mean alone is a very poor temporal characterization of a fracture process and it is suitable to consider the probability density $p_{M_{t h}}(\tau)$ of waiting times $\tau$,

$$
p_{M_{t h}}(\tau) \equiv \frac{\operatorname{Prob}[\tau<\text { waiting time } \leq \tau+d \tau]}{d \tau},
$$

where only events with magnitude $M \geq M_{t h}$ are taken into account.
The probability being invariant under change of variables, $p(T) d T=p(\theta) d \theta$, a transformation of the time axis, $\tau \rightarrow \theta$, changes also the units of the density.

By an optimization procedure, we will verify in the case under study that, for the particular transformation $\tau$ $\rightarrow \tau /\langle\tau\rangle_{M_{t h}}$, all the probability densities of the normalized AE waiting times, $\tau /\langle\tau\rangle_{M_{t h}}$, collapse onto a single curve $p$ implying that we can write the scaling law

$$
p_{M_{t h}}(\tau)=p\left(\tau /\langle\tau\rangle_{M_{t h}}\right) /\langle\tau\rangle_{M_{t h}},
$$

where the scaling function $p\left(\tau /\langle\tau\rangle_{M_{t h}}\right)$ does not depend on $M_{t h}$. The scaling law in Eq. (4) can be obtained also by scaling arguments [15].

Inserting Eq. (2), Eq. (4) takes the form

$$
p_{M_{t h}}(\tau)=10^{-b M_{t h}} \tilde{p}\left(10^{-b M_{t h}} \tau\right),
$$

where $\tilde{p}\left(10^{-b M_{t h}} \tau\right) \equiv k p\left(k 10^{-b M_{t h}} \tau\right)$ and $k \equiv T^{-1} 10^{a}$.

Now, we consider events separated by waiting times $\tau$ for $M \geq M_{t h}$ and $\tau^{\prime} \equiv 10^{b} \tau$ for $M \geq M_{t h}^{\prime} \equiv M_{t h}+1$, and insert these particular arguments, $\left(\tau^{\prime} ; M_{t h}^{\prime}\right)$ and $\left(\tau, M_{t h}\right)$, into Eq. (5). Thus, we obtain

$$
p_{M_{t h}}(\tau)=10^{b} p_{M_{t h}+1}\left(10^{b} \tau\right) .
$$

As shown in Eq. (6), the waiting-time distribution, $p_{M_{t h}}(\tau)$, for different threshold magnitudes, $M_{t h}$, are referred to as being self-similar since they can be obtained from each other by a similarity transformation. For example, the number of AE events with magnitude $M \geq 3.3$ and separated by a waiting-time $\tau=100 \mathrm{~s}$ is about $1 / 10^{b}$ of the number of events with $M \geq 2.3$ and separated by $\tau / 10^{b}$. This is a new tool to describe the temporal evolution of damage phenomena, while the GR law only states that the total number of events with $M \geq 3.3$ is about $1 / 10^{b}$ of the number of events with $M \geq 2.3$ in the whole period under consideration.

\section{EXPERIMENTAL RESULTS}

We apply the above-described analysis to the $\mathrm{AE}$ data from a $1000 \times 150 \times 150 \mathrm{~mm}^{3}$ FRC beam loaded up to failure according to the three-point bending test geometry. The beam had a fiber content of $40 \mathrm{~kg} / \mathrm{m}^{3}$ for a resulting Young's modulus of $35 \mathrm{GPa}$ and a central $50 \mathrm{~mm}$ notch to ensure the development of a central crack during the test, performed with constant displacement rate of $10^{-3} \mathrm{~mm} / \mathrm{s}$ [Fig. 2(a)]. We used an array of AE transducers which exploit the capability of piezoelectric (PZT) crystals to transform elastic vibrations into electric signals. These transducers, resonant in the range of 50 to $800 \mathrm{kHz}$, are designed with detection of fracture precursors, i.e., high-frequency $\mathrm{AE}$, in mind.

After setting an appropriate detection threshold in the AE acquisition system, we verified that no signals were detected before the beginning of the test (i.e., no events without cracks). From each signal we recorded the arrival time, determined with accuracy of about $0.5 \mu \mathrm{s}$, and the amplitude, i.e., the peak voltage of the signal itself. Although a calibration diagram fulfilling metrological requirements of the 


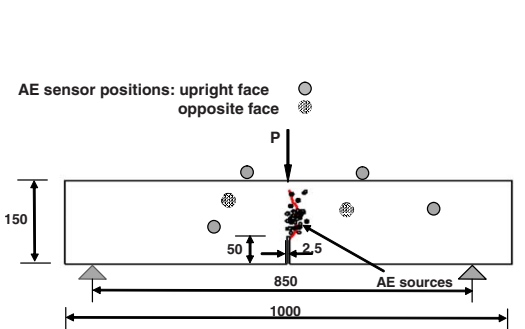

(a)

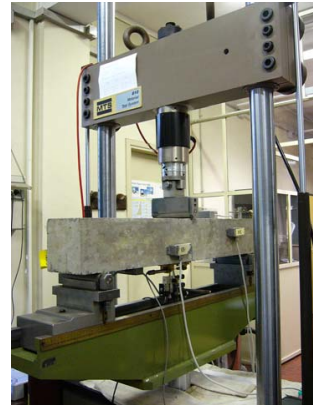

(b)

FIG. 2. (Color online) (a) Three-point bending test geometry, sensor positions, and identification of the fracture by the localized AE sources (black points). (b) Test rig and instrumented beam in the laboratory.

adopted PZT transducers has yet to be determined, the electrical signal can be assumed to be proportional to the acceleration at the specimen surface over the considered bandwidth, and thus be used to quantify signal amplitude.

Six transducers were applied to the specimen at the points shown in Figs. 2(a) and 2(b). The AE source location procedure allowed to identify the fracture process zone developed during the loading test. In this way, AE clusters were seen to propagate with increasing load, following satisfactorily the growth of the central crack [see Fig. 2(a)].

We consider the waiting-time probability densities $p_{M_{t h}}(\tau)$ of the AE events for three values of threshold magnitude $M_{t h} \equiv \log _{10} V_{t h}$, given by the selected amplitude thresholds $V_{t h}=200,400$, and $800 \mu \mathrm{V}$. Fitting the AE data with the GR law yields $b \approx 0.57$, as shown in Fig. 3(a).

Here, we reobtain the scaling law put in the form of Eq. (5) by means of an optimization procedure which leads to the best collapse of the waiting-time distributions in Fig. 4(a). To this purpose, we perform the transformation $\tau \rightarrow 10^{-\alpha M_{t h}} \tau$ and $p_{M_{t h}} \rightarrow 10^{\beta M_{t h}} p_{M_{t h}}$, where $\alpha$ and $\beta$ are the scaling parameters. The optimized parameters, $\alpha=0.60 \pm 0.11$ and $\beta$ $=0.61 \pm 0.11$, yield the collapse of the distributions onto a single curve $p$ shown in Fig. 4(b), and are statistically indistinguishable from the GR $b$ value, $b \approx 0.57$, confirming the validity of Eq. (5).

The scaling function $p(\theta)$ can be well approximated by a generalized gamma function,
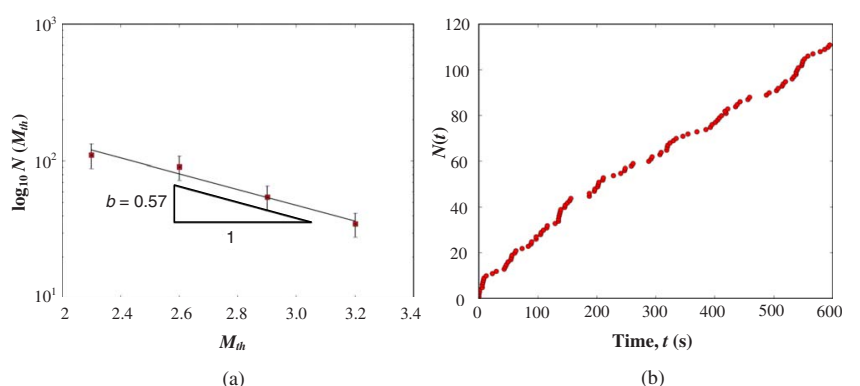

(b)

FIG. 3. (Color online) (a) GR diagram of the number of AEs as a function of their magnitude $M_{t h}\left(M_{t h}=\log _{10} V_{t h}\right.$ with $V_{t h}=200$, $400,800$, and $1600 \mu \mathrm{V})$; the fitting line represents the GR law, $N\left(M_{t h}\right)=10^{a-b M_{t h}}$ with $b \approx 0.57$. (b) Accumulated number $N$ of AEs as a function of time.

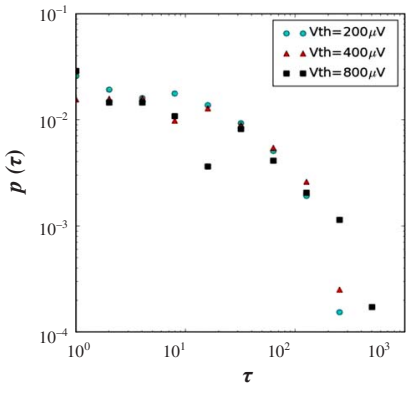

(a)

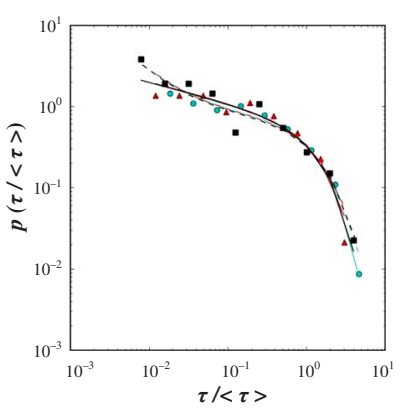

(b)
FIG. 4. (Color online) (a) Probability densities of AE waiting times $\tau$ (measured in seconds) from the experiment. (b) Probability densities of the normalized waiting times $\theta \equiv \tau /\langle\tau\rangle$; the empirical fitting function (solid line) defined in Eq. (7) is compared with the theoretical function (dashed line) defined in [23].

$$
p(\theta) \propto \theta^{-(1-\gamma)} \exp \left[(-\theta / x)^{n}\right],
$$

where $\theta$ is the normalized waiting time, $\theta \equiv \tau /\langle\tau\rangle$. The fit yields $\gamma=0.73 \pm 0.12, \quad x=1.45 \pm 1.26$, and $n=1.24 \pm 0.41$ [13], in good agreement with recent findings for earthquakes $(\gamma \approx 0.7, x \approx 1.5$, and $n \approx 1$ in Ref. [15]) and AEs in rock samples $(\gamma \approx 0.8, x \approx 1.4$, and $n \approx 1$ in Ref. [16]) during stationary periods, i.e., periods where the instantaneous activity rate at any time is statistically indistinguishable from the mean activity rate of the whole period. In fact, the observed data collapses indicate the validity of a scaling law, but the scaling function $f$ generally depends on the considered fracture system and the window of observation. A general (also referred to as universal) scaling function, with a more robust power-law exponent $1-\gamma \cong 0.3$, can be established restricting the analysis to periods of stationary or nearly stationary activity [15].

This claim is confirmed here, where an exponent close to 0.3 is associated with the nearly stationary sequence of $\mathrm{AE}$ events - accumulated number of AEs vs $t$ diagram roughly linear, without abrupt increments - displayed in Fig. 3(b).

However, the supposed existence of a universal scaling law for the waiting-time probability densities was recently criticized. Under very weak and general conditions, Molchan showed mathematically that universality implies that the only possible form for $p\left(\tau /\langle\tau\rangle_{M_{t h}}\right)$ is an exponential function [22], in strong disagreement with observations.

The Molchan argument was generalized by Saichev and Sornette [23,24] and Sornette et al. [25], who developed a theory of the statistics of waiting times in the framework of the epidemic-type aftershock sequence (ETAS) model of triggered seismicity $[26,27]$. They derived a theoretical expression for $p\left(\tau /\langle\tau\rangle_{M_{t h}}\right)$, which essentially depends on the threshold magnitude $M_{t h}$, the size of the studied region, and the tectonic context, apparently in strong disagreement with universality. However, this expression proves to be weakly dependent on these catalog-dependent parameters and statistically identical to empirical expression (7), suggesting that universality is only an approximation, but satisfactory for all practical purposes. Figure 4(b) shows that the theoretical function, with the same parameter values given in [23], fits 
Eq. (7) remarkably well for normalized waiting times $\theta$ $\equiv \tau /\langle\tau\rangle_{M_{t h}}$ larger than about $10^{-2}$. Theoretical and empirical expressions depart from each other at waiting times smaller than $10^{-2}$, in agreement with [23].

\section{NUMERICAL SIMULATIONS}

Here, we perform numerical simulations of the experiment in order to capture the most important characteristics of damage progression and AE activity, verifying in particular the waiting-time scaling properties of the simulated $\mathrm{AE}$ events.

\section{Model}

The numerical procedure is based on a FBM [28] approach, using a Weibull distribution for the local strengths, in which also energy dissipation terms due to crack surface growth are taken into account [29].

More specifically, the specimen of length $L$ is discretized in a "chain of bundles," i.e., an arrangement of $N_{x} \times N_{y}$ "fibers," each identified by the index pair $(i, j)$, with $i$ $=1, \ldots, N_{x}$ and $j=1, \ldots, N_{y}$. Each fiber corresponds to a material portion of length $l$, cross section $S$, and $k=E S / l$, where $E$ is the material Young's modulus. For a given applied center load $P$, the bending stresses $\sigma_{i j}$ in each material portion can be analytically computed,

$$
\sigma_{i j}=-\frac{P x_{i} y_{j}}{2 I_{i}}
$$

where $x_{i}$ and $y_{j}$ are the local (discrete) coordinates and $I_{i}$ the moment of inertia of the $i$ th material section,

$$
I_{i}=\sum_{j} I_{i j}=\sum_{j}\left[b \frac{\left(h / N_{y}\right)^{3}}{12}+\left(y_{j}-y_{C i}\right)^{2} S\right] \text {, }
$$

$h$ and $b$ are the specimen height and depth, respectively, and $y_{C i}$ is the $y$ coordinate of the centroid of the $i$ th section. The tensile strengths of the material portions, $\sigma_{T i j}$, are chosen to be distributed according to a Weibull distribution [30],

$$
P\left(\sigma_{T i j}\right)=1-e^{\left[-\left(\sigma_{T i j} / \sigma_{T}\right)^{m}\right]},
$$

where $\sigma_{T}$ is the characteristic tensile strength of the material and $m$ is the Weibull modulus of the distribution (a variable parameter). Similar behavior is assumed for the compressive strengths $\sigma_{C i j}$. An AE event occurs when the local stress exceeds the compressive or tensile strength and a fiber fails,

$$
\sigma_{i j}<\sigma_{C i j} \text { or } \quad \sigma_{i j}>\sigma_{T i j} .
$$

In this case, the local rigidity $k$ and the moment of inertia $I_{i j}$ are set to zero, and the load is redistributed uniformly among the remaining intact fibers in the $i$ th bundle (this approximation is known as an equal-load-sharing FBM [28]). The amplitude of the AE event is given by the square root of the kinetic energy released in a stress wave $\Delta T_{i j}$, expressed as the difference of the potential-energy variation in the system, $\Delta U_{i j}$, to the dissipated energy in the formation of a new crack surface [31-34],

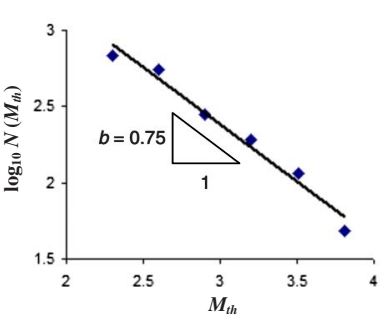

(a)

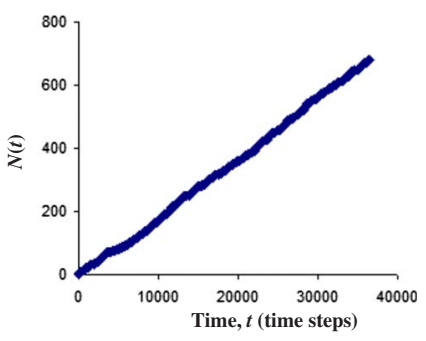

(b)
FIG. 5. (Color online) AE event amplitude distribution obtained in the simulated three-point bending experiment, shown in log-log plot. (a) The number of AEs is plotted as a function of magnitude $M_{t h}\left(M_{t h}=\log _{10} V_{t h}\right.$ with $V_{t h}=200,400,800,1600,3200$, and $6400 \mu \mathrm{V}$ ); the fit yields a GR value of $b \approx 0.75$. (b) Accumulated number of simulated $\mathrm{AE}$ events $\left(N_{\mathrm{AE}}\right)$ vs time (measured in computational steps).

$$
\Delta T_{i j}(t)=\Delta U_{i j}(t)-G_{C} S,
$$

where $G_{C}$ is the critical strain energy release rate of the material.

\section{NUMERICAL RESULTS AND ANALYSIS}

Simulations are carried out on a specimen of dimensions $L=850 \mathrm{~mm}, h=150 \mathrm{~mm}, b=150 \mathrm{~mm}$, Young's modulus $E$ $=48 \mathrm{GPa}$, and tensile and compressive strengths $\sigma_{T}$ $=30 \mathrm{MPa}$ and $\sigma_{C}=45 \mathrm{MPa}$, respectively. The specimen has a central notch of depth $h_{n}=50 \mathrm{~mm}$ and width $l_{n}=2.5 \mathrm{~mm}$, as in the experiment (see Sec. III).

Figure 5(b) shows the number of AE events generated during a load-controlled simulation. The increase in the number of events $N_{\mathrm{AE}}$ is approximately linear in time, and the statistical analysis is carried out on the obtained $661 \mathrm{AE}$ events.

The "cracking pattern" calculated just before failure is shown in Fig. 6, and it can be compared to the real cracking pattern traced over by the localized AE sources (see Fig. 3). This is useful to determine an appropriate value for the Weibull modulus $m$ (this parameter controls the size of the cluster of the AE source points around the notch: the greater $m$, the greater the size; $m=3.5$ is chosen). As in the experiment, the events occur mainly in the central section of the specimen, close to the notch where tensile stresses are the greatest.

In order to compare numerical data with experimental results, the simulated $\mathrm{AE}$ amplitudes are rescaled so that the

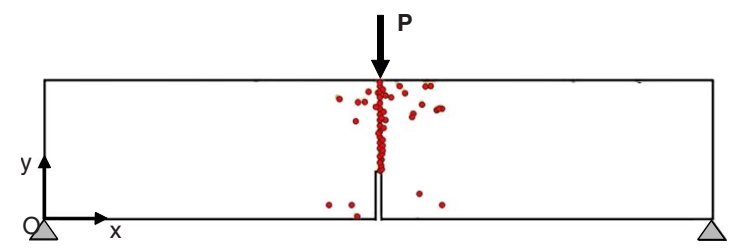

FIG. 6. (Color online) Damage localization in the simulated three-point bending experiment: points indicate damaged portions, i.e., sources of AE. 

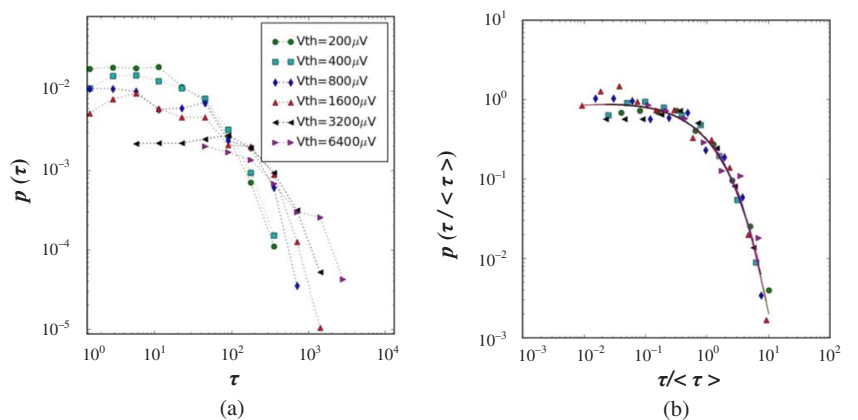

FIG. 7. (Color online) (a) Probability densities of waiting times $\tau$ from the numerical simulation. (b) Probability densities of the normalized waiting times $\tau /\langle\tau\rangle$; the fit is an exponential function.

maximum experimental and numerical values coincide, and numerical AE amplitudes are also expressed in microvolts. Clearly, this rescaling does not influence the amplitude distribution statistical analysis. The AE amplitude distribution follows the GR law with $b \approx 0.75$, as shown in Fig. 5(a). Data analysis is now analogous to that carried out in the case of experimental measurements: the waiting-time distributions for the simulated AE events [shown in Fig. 7(a)] can be determined for various amplitude thresholds, ranging from 200 to $6400 \mu \mathrm{V}$. The same distributions collapse onto a single curve after rescaling, as shown in Fig. 7(b).

The optimized scaling parameters, $\alpha=0.71 \pm 0.04$ and $\beta$ $=0.71 \pm 0.04$, are statistically compatible with the GR $b$ value $(b \approx 0.75)$ shown in Fig. $5(\mathrm{a})$, confirming in this way the scaling law of Eq. (5). A fit yields the parameter values $\gamma=1.06 \pm 0.17, x=0.63 \pm 0.59$, and $n=0.68 \pm 0.15$, implying a power-law exponent $1-\gamma \approx 0$ and thus a near-exponential scaling function, which describes an almost memoryless (Poisson) process [Fig. 7(b)]. Therefore, numerical simulations correctly reproduce the spatial and size distributions of microfractures, but do not capture the dynamics of the damage process since springs break in a nearly stochastic manner in time, while the power-law decay of experimental distributions indicates clustering of microfractures in time. This behavior is indicative of a low-correlated system [35], as ex- pected from the chosen FBM: the rupture of a single spring only influences the remaining springs in the same material section, where the load is redistributed, and stress concentrations are small because an equal load sharing model is adopted [35]. It is expected that models with a higher degree of correlation among fracturing elements yield higher clustering in time, and therefore a steeper power law.

\section{CONCLUSIONS}

The data collapse of AE waiting-time distributions onto a scaling function is here verified both in a laboratory and a simulated fracture experiment. The scaling law indicates the self-similarity of the waiting-time distributions at different magnitude levels, which allows to capture more information about the dynamics of the damage process.

This analysis can highlight substantial qualitative differences between apparently similar data sets, in this case the experimental and numerical results: the latter, generated using a FBM, display the behavior of low-correlated systems (as one would expect for earthquakes occurring on different fault lines), for which little clustering occurs in the timing of microfractures. Though data collapse occurs in both cases using the chosen rescaling procedure, the waiting-time distributions from the laboratory test and the numerical simulation are described by two different scaling laws, i.e., a gamma and a Poisson distribution, respectively. The powerlaw decay in the scaling function obtained for the experimental data indicates a clustering behavior in which more events are separated by shorter waiting times than in a random memoryless occurrence, e.g., a Poisson process with the same rate, as in the numerical data. It is expected that the damage dynamics could be numerically simulated in a more realistic way using a model with a greater degree of longrange correlation between fracturing elements. The use of lattice models looks promising since the latter provide a more faithful description of the microstructural texture and the stress pattern in engineering materials than FBMs do. This will be the object of future investigations.
[1] D. L. Turcotte, W. I. Newman, and R. Shcherbakov, Geophys. J. Int. 152, 718 (2003).

[2] L. M. Kachanov, Introduction to Continuum Damage Mechanics (Martinus Nijhoff, Dordrecht, The Netherlands, 1986).

[3] J. Lemaitre and J. L. Chaboche, Mechanics of Solid Materials (Cambridge University Press, Cambridge, 1990).

[4] D. Krajcinovic, Damage Mechanics (Elsevier, Amsterdam, 1996).

[5] A. Carpinteri, G. Lacidogna, G. Niccolini, and S. Puzzi, Meccanica 43, 349 (2008).

[6] A. Guarino, A. Garcimartin, and S. Ciliberto, Eur. Phys. J. B 6, 13 (1998).

[7] D. Lockner, Int. J. Rock Mech. Min. Sci. Geomech. Abstr. 30, 883 (1993).

[8] A. Carpinteri, G. Lacidogna, G. Niccolini, and S. Puzzi, Int. J.
Damage Mech. 18, 259 (2009).

[9] S. Colombo, I. G. Main, and M. C. Forde, J. Mater. Civ. Eng. 15, 280 (2003).

[10] A. Saichev and D. Sornette, Phys. Rev. E 70, 046123 (2004).

[11] H. E. Stanley, Rev. Mod. Phys. 71, S358 (1999).

[12] P. Bak, K. Christensen, L. Danon, and T. Scanlon, Phys. Rev. Lett. 88, 178501 (2002).

[13] G. Niccolini, G. Durin, A. Carpinteri, G. Lacidogna, and A. Manuello, J. Stat. Mech.: Theory Exp. 2009, P01023.

[14] A. Corral, in Modelling Critical and Catastrophic Phenomena in Geoscience: A Statistical Physics Approach, edited by P. Bhattacharyya and B. K. Chakrabarti, Lecture Notes in Physics No. 705 (Springer, Berlin, 2006), pp. 191-221.

[15] A. Corral, in Acoustic Emission and Critical Phenomena: From Structural Mechanics to Geophysics, edited by A. 
Carpinteri and G. Lacidogna (Taylor \& Francis, London, 2008).

[16] J. Davidsen, S. Stantchis, and G. Dresen, Phys. Rev. Lett. 98, 125502 (2007).

[17] K. Mogi, Bull. Earthquake Res. Inst., Univ. Tokyo 40, 831 (1962).

[18] C. H. Scholz, Bull. Seismol. Soc. Am. 58, 399 (1968).

[19] M. C. Miguel, A. Vespignani, S. Zapperi, J. Weiss, and J. R. Grasso, Nature (London) 410, 667 (2001).

[20] C. F. Richter, Elementary Seismology (Freeman, San Francisco, 1958).

[21] M. V. M. S. Rao and K. J. P. Lakshmi, Curr. Sci. 89, 1577 (2005).

[22] G. M. Molchan, Pure Appl. Geophys. 162, 1135 (2005).

[23] A. Saichev and D. Sornette, Phys. Rev. Lett. 97, 078501 (2006).

[24] A. Saichev and D. Sornette, J. Geophys. Res. 112, B04313 (2007).
[25] D. Sornette, S. Utkin, and A. Saichev, Phys. Rev. E 77, 066109 (2008)

[26] Y. Y. Kagan and L. Knopoff, J. Geophys. Res. 86, 2853 (1981).

[27] A. Helmstetter and D. Sornette, J. Geophys. Res. 107, 2237 (2002).

[28] F. Kun, S. Zapperi, and H. J. Hermann, Eur. Phys. J. B 17, 269 (2000).

[29] N. Pugno, J. Phys.: Condens. Matter 18, S1971 (2006).

[30] W. Weibull, Proc. R. Swed. Inst., Eng. Res. 151, 1 (1939).

[31] F. Bosia, N. Pugno, G. Lacidogna, and A. Carpinteri, Int. J. Solids Struct. 45, 5856 (2008).

[32] N. Pugno, A. Carpinteri, P. P. Delsanto, F. Bosia, and A. Gliozzi, Phys. Rev. E 78, 046103 (2008).

[33] N. Pugno, F. Bosia, and A. Carpinteri, Small 4, 1044 (2008).

[34] A. Carpinteri and N. Pugno, J. Appl. Mech. 69, 854 (2002).

[35] S. Pradhan and A. Hansen, Phys. Rev. E 72, 026111 (2005). 